\title{
The Relationship Between Thyroid Function and T Lymphocyte Subsets in Patients with Hashimoto's Thyroiditis
}

\author{
Rongqin Dai ${ }^{1}$, Jinbiao Zhang ${ }^{2}{ }^{*}$, Wei $\mathrm{Li}^{2}$, Xiangxing $\mathrm{Lu}^{2}$, Wenyan $\operatorname{Tian}^{2}$ \\ ${ }^{1}$ Institute of Thyroid Disease Affiliated to Cangzhou Medical College, Cangzhou, China \\ ${ }^{2}$ Cangzhou Hospital of Integrated Traditional Chinese and Western Medicine, Cangzhou, China
}

Email address:

zjb7829325@163.com (Jinbiao Zhang)

${ }^{*}$ Corresponding author

To cite this article:

Rongqin Dai, Jinbiao Zhang, Wei Li, Xiangxing Lu, Wenyan Tian. The Relationship Between Thyroid Function and T Lymphocyte Subsets in Patients with Hashimoto's Thyroiditis. American Journal of Biomedical and Life Sciences. Vol. 8, No. 4, 2020, pp. 114-118. doi: $10.11648 /$ j.ajbls.20200804.17

Received: July 16, 2020; Accepted: August 14, 2020; Published: August 25, 2020

\begin{abstract}
Objective: To assess the relationship between thyroid function and $\mathrm{T}$ lymphocyte subsets in Patients with Hashimoto's Thyroiditis. Method: To select 131 diagnosed HT patients as the research object, which is divided into HT hyperthyroidism group (Group A) with 18 cases, normal HT thyroid function group (Group B) with 35 cases, HT subclinical hypothyroidism group (Group C) with 42 cases, and HT hypothyroidism group (Group D) with 36 cases in accordance with thyroid function. Besides, 40 cases of healthy check-up people are selected as the control group. ECLIA is adopted to test the thyroid function index of each group, and FCM is adopted to test T lymphocyte subsets. Result: Compare $\mathrm{CD} 3^{+} \mathrm{T}$ lymphocytes of each group, Group D is obviously higher than Group A, Group B, and the control group, and the difference has statistic significance $(\mathrm{P}<0.05)$; Compare $\mathrm{CD} 3^{+} \mathrm{CD} 4^{+} \mathrm{T}$ lymphocyte and $\mathrm{CD} 4^{+} / \mathrm{CD} 8^{+}$of each group, Group $\mathrm{D}$ is obviously higher than other groups, Group C is obviously higher than Group A, Group B, and the control group, Group A and Group B are obviously higher than the control group, and the differences have statistic significance $(\mathrm{P}<0.05)$; Compare $\mathrm{CD}^{+} \mathrm{CD} 8^{+} \mathrm{T}$ lymphocytes of each group, Group D is obviously lower than other groups, Group C is obviously lower than Group A, Group B, and the control group, the difference has statistic significance $(\mathrm{P}<0.05)$. The correlation analysis shows that TSH is in positive correlation with $\mathrm{CD}^{+} / \mathrm{CD}^{+}, \mathrm{FT} 3$ is in positive correlation with $\mathrm{CD}^{+} \mathrm{CD}^{+} \mathrm{T}$, and $\mathrm{FT} 4$ is in positive correlation with $\mathrm{CD} 3^{+} \mathrm{CD} 8^{+} \mathrm{T}$ and in negative correlation with $\mathrm{CD}^{+} / \mathrm{CD}^{+}$. Conclusion: HT patients have the imbalance of $\mathrm{T}$ lymphocyte subsets, and the $\mathrm{T}$ lymphocyte subsets ratio of HT patients of different thyroid function has obvious differences, which may have close relationship with the occurrence and development of HT.
\end{abstract}

Keywords: Hashimoto's Thyroiditis, Thyroid Function, T Lymphocyte Subsets

\section{Introduction}

Hashimoto's Thyroiditis (HT) is an autoimmune thyroid disease of organ specificity mediated by $\mathrm{T}$ cells. Its pathogenesis is complicated, and it is deemed that the disorder of body immune function is caused by the mutual function among inheritance, environment, etc., the immune tolerance is destroyed, auto-reactive $\mathrm{T}$ cells are activated [1], suppressor T cells allow auxiliary $\mathrm{T}$ cells to react with the antigens under a certain condition, which causes the damage of thyroid cells [2], and then the disorder of thyroid function in return. In the development process of the disease, the patients' thyroid function can be manifested as normal thyroid function or transient hyperthyroidism. With the continuous thyroid cell damage, it is finally manifested as hypothyroidism [3]. Previous studies prove that the abnormal activation of auto-reactive $\mathrm{T}$ cells has close relationship with the HT occurrence, and participates in the attack process of HT [4]. There are relatively few studies on the peripheral blood $\mathrm{T}$ lymphocyte subsets change condition of HT patients of 
different thyroid function. By analyzing the peripheral blood $\mathrm{T}$ lymphocyte subsets change condition of HT patients of different thyroid function, this paper aims at further studying the role of T lymphocytes in HT occurrence and development.

\section{Methods}

\subsection{General Information}

131 patients who were clinically diagnosed with HT in the endocrinology department of Cangzhou Hospital of Integrated Traditional Chinese and Western Medicine, Hebei province from December, 2017 to February, 2019 were chosen to be study objects, among which there were 26 men cases, 105 women cases with age from $20 \sim 68$, and the average age were $(44.57 \pm 16.76)$ years old. They were divided into Hashimoto hyperthyroidism group (Group A)within 18 cases, normal Hashimoto's thyroid function group (Group B) within 35 cases, Hashimoto Subclinical hypothyroidism group (Group C)within 42 cases and Hashimoto hypothyroidism group (Group D) within 36 cases and on the basis of Thyroid function. Besides, 40 cases of healthy check-up people are selected as the control group, in which there were 8 men cases and 32 women cases with age from $22 \sim 67$, and the average age were $(45.33 \pm 16.48)$ years old. The general clinical data such as gender and age were compared among groups, and the differences have no statistical significance. This research has been approved by the ethics committee and all the subjects were informed and agreed.

\subsection{Diagnostic Criteria and Exclusion Criteria}

\subsubsection{Diagnostic Criteria of Hashimoto's Thyroiditis}

The HT diagnosis refers to the diagnostic criteria in the 8th version of Internal Medicine published by the People's medical publishing house [5], including: (1) The one with clinical features, such as diffusing goiter, tough texture, especially with thickening of isthmus and pyramidal lobe, etc., and Thyroid peroxidase antibody (TPOAb) or Thyroglobulin antibody (TGAb) positive; (2) The one without goiter, but with distinct TPOAb or TGAb increase as well as hypothyroidism.

\subsubsection{Diagnostic Criteria of Thyroid Dysfunction}

The diagnostic criteria of thyroid dysfunction including [6]: (1)Hyperthyroidism: TSH $<0.27 \mathrm{mU} / \mathrm{L}$, FT3 $>7.1 \mathrm{pmol} / \mathrm{L}$, FT4 $>22$ pmol/L; (2) Subclinical hypothyroidism: TSH $>4.2$ $\mathrm{mU} / \mathrm{L}, \mathrm{FT} 3$ and FT4 are normal; (3) Hypothyroidism: TSH $>$ $4.2 \mathrm{mU} / \mathrm{L}, \mathrm{FT} 3<2.8 \mathrm{pmol} / \mathrm{L}$ and / or FT4 $<12 \mathrm{pmol} / \mathrm{L}$.

\subsubsection{Exclusion Criteria}

(1) Patient with abnormal liver and kidney function, and/or cardiac insufficiency; (2) The pregnant or breastfeeding woman; (3) Patient with autoimmune diseases; (4) With the history of pituitary or hypothalamus disease; (5) Taked medicine which affects thyroid function.

\subsection{Experimental Method}

\subsubsection{Specimen Collection}

Morning fasting venous blood is taken from all the subjects and put into pro-coagulation tube and EDTA-K2 anticoagulant tube. Serum is separated from the pro-coagulation tube timely for testing thyroid function. The specimen in the EDTA-K2 anticoagulant tube is shaken evenly, without centrifugation, for testing $\mathrm{T}$ lymphocyte subsets with the whole blood.

\subsubsection{Thyroid Function and Antibody Test}

Adopt Cobas E601 electrogenerated chemiluminescence analyzer of Roche supporting assay to test thyroid function and antibody indicator, including TSH, FT3, FT4, TT3, TT4, $\mathrm{TPOAb}$ and TGAb.

\subsubsection{The Testing of T Lymphocyte Subsets}

Flowcytometry FACSCalibur provided by American BD company is adopted to test $\mathrm{T}$ lymphocyte subsets, including the percentage of $\mathrm{CD}^{+} \mathrm{T}, \mathrm{CD}^{+} \mathrm{CD}^{+} \mathrm{T}, \mathrm{CD} 3^{+} \mathrm{CD} 8^{+} \mathrm{T}$ and $\mathrm{CD} 4^{+} / \mathrm{CD}^{+}$. All the reagents are corollary reagents of instrument companies.

\subsection{Statistic Method}

The data adopts SPSS24.0 software for analysis and processing, the metering data is expressed by $(\bar{x} \pm \mathrm{s})$, the comparison among multiple groups adopts analysis of variance, pairwise comparison adopts SNK-q test, and correlation analysis adopts linear correlation analysis.

\section{Results}

\subsection{The Comparison of Each Group of T Lymphocyte Subsets}

Compare $\mathrm{CD}^{+} \mathrm{T}$ lymphocytes of each group, Group D is obviously higher than Group A, Group B, and the control group, and the difference has statistic significance $(\mathrm{P}<0.05)$, the comparison of other groups has no statistic significance ( $\mathrm{P}>0.05$ ); Compare $\mathrm{CD} 3{ }^{+} \mathrm{CD} 4^{+} \mathrm{T}$ lymphocyte and $\mathrm{CD} 4^{+} / \mathrm{CD} 8^{+}$ of each group, Group D is obviously higher than other groups, Group C is obviously higher than Group A, Group B, and the control group, Group A and Group B are obviously higher than the control group, and the differences have statistic significance $(\mathrm{P}<0.05)$, as for the comparison of Group A and Group B, the difference has no statistic significance $(\mathrm{P}>0.05)$; Compare $\mathrm{CD}^{+} \mathrm{CD}^{+} \mathrm{T}$ lymphocytes of each group, Group D is obviously lower than other groups, Group $\mathrm{C}$ is obviously lower than Group A, Group B, and the control group, the difference has statistic significance $(\mathrm{P}<0.05)$, as for the comparison of Group A and Group B with the control group, the difference has no statistic significance $(\mathrm{P}>0.05)$. (Table 1) 
Table 1. Comparison of T lymphocyte subsets in each group $\left(\bar{x}_{ \pm s}\right)$.

\begin{tabular}{llllll}
\hline Groups & $\mathbf{n}$ & $\mathbf{C D 3}^{+} \mathbf{T}$ & $\mathbf{C D 3}^{+} \mathbf{C D} 4^{+} \mathbf{T}$ & $\mathbf{C D 3}^{+} \mathbf{C D 8}^{+} \mathbf{T}$ & $\mathbf{C D 4}^{+} / \mathbf{C D 8}^{+}$ \\
\hline normal control group & 40 & $68.73 \pm 9.72$ & $40.14 \pm 9.34$ & $27.03 \pm 5.83$ & $1.58 \pm 0.62$ \\
group A & 18 & $68.84 \pm 9.45$ & $42.07 \pm 8.14$ & $26.66 \pm 6.14$ & $1.62 \pm 0.73$ \\
group B & 35 & $68.79 \pm 9.67$ & $42.54 \pm 8.43$ & $26.32 \pm 6.72$ & $1.68 \pm 0.63$ \\
group C & 42 & $69.85 \pm 8.12$ & $44.18 \pm 8.82$ & $25.16 \pm 6.63$ & $1.85 \pm 0.89$ \\
group D & 36 & $71.17 \pm 8.10$ & $46.82 \pm 8.89$ & $23.48 \pm 7.01$ & $2.01 \pm 0.97$ \\
F & & 5.13 & 8.11 & 6.36 & 7.65 \\
$P$ & & 0.047 & 0.039 & 0.043 & 0.037 \\
\hline
\end{tabular}

\subsection{The Correlation Between Thyroid Function Index and T Lymphocyte Subsets}

The correlation analysis shows that TSH is in positive correlation with $\mathrm{CD}^{+} / \mathrm{CD}^{+}$, FT3 is in positive correlation with $\mathrm{CD}^{+} \mathrm{CD}^{+} \mathrm{T}$, and $\mathrm{FT} 4$ is in positive correlation with $\mathrm{CD}^{+} \mathrm{CD}^{+} \mathrm{T}$ and in negative correlation with $\mathrm{CD} 4^{+} / \mathrm{CD}^{+}$. (Table 2)

Table 2. The correlation between thyroid function index and T lymphocyte subsets.

\begin{tabular}{lllll}
\hline thyroid function & $\mathbf{r}$ & & & \\
\hline index & $\mathbf{C D 3}^{+} \mathbf{T}$ & $\mathbf{C D 3}^{+} \mathbf{C D 4}^{+} \mathbf{T}$ & $\mathbf{C D 3}^{+} \mathbf{C D 8}^{+} \mathbf{T}$ & $\mathbf{C D 4}^{+} / \mathbf{C D 8}^{+}$ \\
\hline TSH & 0.169 & 0.354 & 0.412 & $0.572^{*}$ \\
FT3 & 0.201 & 0.231 & $0.628^{*}$ & 0.312 \\
TT3 & 0.067 & 0.123 & 0.446 & 0.279 \\
FT4 & 0.278 & 0.404 & $0.649^{*}$ & $-0.478^{*}$ \\
TT4 & 0.015 & 0.257 & 0.368 & 0.157 \\
\hline
\end{tabular}

Note: $* \mathrm{P}<0.05$.

\section{Discussions}

Hashimoto's Thyroiditis is a common autoimmune thyroid disease of organ specificity mediated by T cells, the abnormal activation of auto-reactive $\mathrm{T}$ cells has close relation with the attack of HT and participates in the attack process of HT. T cell is one kind of important $\mathrm{T}$ lymphocytes in the body, with its main role as mediating cell immune response and regulating body immune function. T cells can be divided into different subsets in accordance with its surface $\mathrm{CD}$ molecules, $\mathrm{CD}^{+} \mathrm{T}$ cells represents the total $\mathrm{T}$ lymphocytes, $\mathrm{CD}^{+} \mathrm{CD} 4^{+} \mathrm{T}$ cells represents helper $\mathrm{T}$ cells $(\mathrm{Th})$, which mainly mediate cell immune response and assist $\mathrm{B}$ cells to produce humoral immune response, $\mathrm{CD}^{+} \mathrm{CD}^{+} \mathrm{T}$ cells include cytotoxic $\mathrm{T}$ cells as well as suppressor $\mathrm{T}$ cells (Ts). The suppressor $\mathrm{T}$ cells mainly suppress the proliferation of self $\mathrm{T}$ lymphocytes to the alloantigen and suppress B lymphocytes to produce antibody. Under normal condition, various lymphocyte subsets of the body maintain at a certain quantity and ratio, interact, and maintain the normal immune function of the body [7].

Studies discover that the change of $\mathrm{T}$ lymphocyte subsets has close relationship with the occurrence and development of HT [8]. Under the joint action of inheritance and environment, the imbalance between Th cells and Ts cells is destroyed. Th cell is an important index of regulating human body's immune function, and has an important role in maintaining the balance of body immune function [9]. By comparing the change condition of HT patients' $\mathrm{T}$ lymphocyte subsets of different thyroid function, the paper discovers that Th cells of each HT group are obviously higher than the control group, the increase of HT hypothyroidism group is the most obvious, and then the HT subclinical hypothyroidism group, which explains that Th cells are increasing continuously with the development of HT patients' disease. Th mainly includes Th1, Th2, and Th17 [10], Th1 cells can secrete IFN- $\gamma$, IL-2 and other cytokines, IFN- $\gamma$ can increase the expression of HLA-II type antigen of thyroid epithelial cell, cause the secretion and release of IL-2, IL-2 can activate and secrete other cytokines and makes a large amount of T lymphocytes and mononuclear cells enter into the thyroid and increase cytotoxicity [11-12]. Studies discover that there is the hyperfunction of Th1 cytokine in the attack process of HT [13]; Th2 cells can promote B cells' activation and antibody production by secreting IL-4, IL-6 and other cytokines, and mediate humoral immune response. Th17 is a cell subset which mainly secretes IL-17, induces the production of several kinds of inflammatory cytokines and chemokines through secreting IL-17 in large amount, invades the tissue cells, and causes the damage of tissue cells [14]. Studies discover that the change of self micro environment within the HT patients can further induce the divergence of Th17 cells [15], the increased Th17 cells can promote the synthesis of relevant cytokines and cause the imbalance of inflammatory cytokines in the body. The imbalance of Th1 and Th17 cells can induce and deteriorate HT, and further affect thyroid function [16].

This paper shows that HT manifests Th cells increase in hyperthyroidism period, while Ts cells reduction starts at the subclinical hypothyroidism stage, which conforms to the conclusion that $\mathrm{Th}$ cells are the main invasive cells at the early stage of the thyroid cells [17]. At the early stage of HT, Th cells have invaded in the thyroid tissue in large amount, the activation of $\mathrm{T}$ lymphocyte and macrophage, as well as the release of various inflammatory cytokines causes the damage of thyroid tissue, which makes the thyroid hormone stored in thyroid follicle cells release into blood in large amount and causes hyperthyroidism in return. For HT patients with normal thyroid function, whose thyroid function index is expressed as normal, but Th cells, TPOAb, TGAb level increase obviously, which explains that immune disorder also exists in HT patients with normal thyroid function, which deserves clinical attention. With the further development of the disease, Th cells within the patient increases continuously, and the Ts cells with suppressing role reduces continuously, the Th function gets active while Ts function has defects, which activates $\mathrm{B}$ cells to produce a 
large amount of self antibody of thyroid, thyroid cells have cell invasive inflammation, a large amount of lymphocytes, NK cells, plasma cells and macrophages invade, which causes damage to thyroid tissue while self antibody of the thyroid combines with corresponding antigen, which further destroys thyroid follicular membrane through the activation of complement and ADCC action and causes hypothyroidism [18-19], and the patients manifest as subclinical hypothyroidism or hypothyroidism. The research shows that hypothyroidism and subclinical hypothyroidism patients'Th cells are higher than that of HT patients with hyperthyroidism or normal thyroid function, while hypothyroidism and subclinical hypothyroidism patients'Ts cells are lower than that of HT patients with hyperthyroidism or normal thyroid function, which explains that the change of $\mathrm{T}$ lymphocyte subsets has close relationship with the seriousness of HT. By observing the correlation between thyroid function index and $\mathrm{T}$ lymphocyte subsets, this paper discovers that TSH is in positive correlation with $\mathrm{CD} 4^{+} / \mathrm{CD}^{+}$, and $\mathrm{FT} 3, \mathrm{FT} 4$ are in positive correlation with $\mathrm{CD}^{+} \mathrm{CD}^{+} \mathrm{T}$, FT4 is in negative correlation with $\mathrm{CD} 4^{+} / \mathrm{CD} 8^{+}$. It can be considered that the influence of $\mathrm{T}$ lymphocyte subsets on thyroid function is the result of joint action of Th and Ts, while hypothyroidism, especially the reduction of FT3, FT4, may deteriorate the imbalance of HT patients' peripheral blood lymphocyte subsets.

\section{Conclusions}

Hashimoto's Thyroiditis patients have the imbalance of $\mathrm{T}$ lymphocyte subsets, which expresses as the hyperfunction of Th cells and the defect of Ts cells. The increase of Th cells may exist in HT patients of different thyroid function, the HT hypothyroidism patient is the most obvious, and then followed by the HT subclinical hypothyroidism patient; the reduction of Ts cells starts from the HT subclinical hypothyroidism patient, while the hypothyroidism patient is the most obvious. The imbalance of $\mathrm{T}$ lymphocyte subsets plays an important role in the occurrence and development of HT patients.

\section{Acknowledgements}

This study was supported by a project grant from: science and technology research and development guidance plan project in cangzhou (Grant No. 172302039).

\section{References}

[1] Ma Qianqian, Liang Qiuhua, Sun Lin, et al. Expression and significance of $\mathrm{CD}^{+} \mathrm{CD} 45 \mathrm{RO}^{+}$memory $\mathrm{T}$ cell in peripheral blood of patients with Hashimoto's thyroiditis [J]. Chinese Journal of Immunology, 2016, 32 (10): 1527-1531.

[2] He Ke, Lu Xiwan, Zhu Lihua, et al. Abnormal proportions of immune regulatory cells in peripheral blood of patients with Hashimoto's Thyroiditis [J]. Chinese Journal of Immunology, 2019, 35 (20): 2513-2516.
[3] Chong Xianjin, Yu Qingyuan, Aman Guli-Suli Tang, et al. The effect of dexamethasone combined with thyroxine in the treatment of autoimmune thyroiditis and its effect on the related serum cytokines and $\mathrm{Th} 1 / \mathrm{Th} 2$ related cytokines [J]. Chinese Journal of Difficult and Complicated Cases, 2018, 17 (11): 1233-1237.

[4] Guo H, Peng D, Wang Y, et al. A higher frequency of circulating IL-22(+)CD4(+) T cells in Chinese patients with newly diagnosed Hashimoto's thyroiditis [J]. PLoS One, 2014, 9 (1): e84545.

[5] Ge Junbo, Xu Yongjian. Internal Medicine [M]. 8th Edition. Beijing: People's Medical Publishing House, 2013: 698.

[6] Dai Rongqin, Liu Yuzhi, Wang Fengling, et al. The incidence of hashimoto's thyroiditis in medical examination population in Huanghua City, Hebei Province [J]. Chinese Journal of Endemiology, 2019, 38 (2): 140-143.

[7] Liang Daoyan, Guo Chunyan, Feng Qing, et al. Effects and changes of retinol-binding protein and $T$ cell subsets in children with mycoplasma pneumoniae infection [J]. Chinese Journal of Laboratory Diagnosis, 2018, 22 (12): 2053-2056.

[8] Wang Xiumin, Dong Yue, Zhao Lei, et al. Clinical effect and influence of combined selenium with soothing liver and activating spleen method on T-lymphocyte subsets in Hashimoto's thyroiditis treatment [J]. Northwest Pharmaceutical Journal, 2017, 32 (6): 779-782.

[9] Jin Xin, Shang Xuemei, Chen Haibo, et al. Study on dynamic changes and significance of the peripheral blood regulatory $\mathrm{T}$ cells (Treg) and Th17 cells in patients with Hashimoto's thyroiditis [J]. Journal of Clinical and Experimental Medicine, 2018, 17 (19): 2092-2094.

[10] Li Ying, Song Yu, Zhang Tianqi, et al. Relative risk of peripheral blood $\mathrm{T}$ lymphocyte subsets variable quantity in psoriasis vulgaris $[\mathrm{J}]$. Chinese Journal of Laboratory Diagnosis, 2019, 23 (7): 1177-1180.

[11] Jin X, Wang S, Zhao X, et al. Coumestrol inhibits autoantibody production through modulating Th1 response in experimental autoimmune thyroiditis [J]. Oncotarget, 2016, 7 (33): 52797-52809.

[12] Sharma R, Di Dalmazi G, Caturegli P. Exacerbation of autoimmune thyroiditis by CTLA-4 Blockade: a role for IFN- $\gamma$ induced indoleamine 2, 3-dioxygenase [J]. Thyroid, 2016, 26 (8): 1117-1124.

[13] Yu Y, Yu N, Lu G, et al. Hashimoto's thyroiditis with elevated serum $\mathrm{IgG}_{4}$ concentrations is not equivalent to $\mathrm{IgG} 4$ Hashimoto's thyroiditis [J]. Clin Endocrinol (Oxf), 2018, 88 (6): 943-949.

[14] Noack M1, Miossec P. Th17 and regulatory T celI balance in autoimmune and inflammatory diseases [J]. Autoimmun Rev, 2014, 13 (6): 668-677.

[15] Lv Yanli. The Value of Conventional Color Doppler Ultrasound Combined with Ultrasound Elastography in Diagnosing Diffuse Goiter (Graves' Disease) and Hashimoto's Thyroiditis (HT) [J]. Chinese Journal of Ultrasound in Medicine, 2013, 29 (11): 961-964.

[16] Chen Baoqin, Li Shi, Zhang Jiawei, et al. Significance of serum TPOAb and TGAb in the diagnosis of Hashimoto's thyroiditis and hyperthyroidism [J]. Chinese Journal of Laboratory Diagnosis, 2015, 19 (11): 1932-1933. 
[17] Suciu-Foca N, Manavalan JS, Scotto L, et a1. Molecular characterization of all specific $\mathrm{T}$ suppressor and tolerogenic dendritic ceils: review [J]. IntImmunopharmacol, 2005, 5 (1): 7-11.

[18] Dai Qing, He Jun, Zhang Fan, et al. Expression of IL-1 $\beta$ in peripheral blood of Hashimoto's thyroiditis and its relationship with TGAb and TPOAb [J]. Chinese Journal of Gerontology, 2018, 38 (7): 1574-1575.
[19] Safdari V, Alijani E, Nemati $M$, et al. Imbalances in $T$ cell-related transcription factors among patients with Hashimoto's thyroiditis [J]. Sultan Qaboos Univ Med J, 2017: 17 (2): e174-180. 